\title{
A Multivariate Stochastic Hybrid Model with Switching Coefficients and Jumps: Solution and Distribution
}

\author{
D. P. Siu and G. S. Ladde \\ Department of Mathematics and Statistics, University of South Florida, Tampa, FL 33620, USA \\ Correspondence should be addressed to G. S. Ladde, gladde@usf.edu
}

Received 9 May 2011; Accepted 7 June 2011

Academic Editor: V. V. Anh

Copyright (C) 2011 D. P. Siu and G. S. Ladde. This is an open access article distributed under the Creative Commons Attribution License, which permits unrestricted use, distribution, and reproduction in any medium, provided the original work is properly cited.

\begin{abstract}
In this work, a class of multidimensional stochastic hybrid dynamic models is studied. The system under investigation is a first-order linear nonhomogeneous system of Itô-Doob type stochastic differential equations with switching coefficients. The switching of the system is governed by a discrete dynamic which is monitored by a non-homogeneous Poisson process. Closed-form solutions of the systems are obtained. Furthermore, the major part of the work is devoted to finding closed-form probability density functions of the solution processes of linear homogeneous and Ornstein-Uhlenbeck type systems with jumps.
\end{abstract}

\section{Introduction}

The study of stochastic hybrid systems exhibiting both continuous and discrete dynamics has been an area of great interest over the years. The properties of various types of stochastic hybrid systems have been studied extensively. Davis [1,2] introduced a piecewisedeterministic Markov process, where transitions between discrete modes are triggered by random events and deterministic conditions for hitting the boundary, while the continuousstate process between jumps for the model is governed by a deterministic differential equation. Hespanha [3] proposed a model where transitions between modes are triggered by stochastic events much like transitions between states of a continuous-time Markov chains. Hu et al. [4] proposed a stochastic hybrid system where the deterministic differential equations for the evolution of the continuous-state process are replaced by Itô-Doob type stochastic differential equations [5,6]. However, in this proposed model, the transitions are only triggered by hitting the boundaries. Siu and Ladde [7] studied a stochastic hybrid 
dynamic process where the transitions of its discrete time state are governed by either a nonhomogeneous Poisson process or triggered by hitting the boundaries, while the continuous state is governed by a stochastic diffusion. A study of a stochastic hybrid system whose continuous time component is stochastic and altered by transitions of a finite-state Markov chain can be found in Chandra and Ladde [8], Korzeniowski and Ladde [9], and Ladde [10]. Yin et al. [11] provided an algorithm of the numerical solutions for a class of jump diffusions with regime switching. Mao and Yuan [12] and Yin and Zhu [13] summarize a wide range of properties of stochastic differential equations with Markovian switching coefficients. In view of applications, stochastic hybrid systems have been employed to diverse fields of studies, such as communication networks [3,14], air traffic management $[15,16]$, and insurance risk models [17].

In this work, we attempt to solve two fundamental problems in the stochastic modeling of dynamic processes described by Itô-Doob type stochastic differential equations with jumps. First, we find closed-form solutions of the stochastic hybrid systems. By using the closed-form solutions, we determine the closed-form probability density functions of solution processes of special cases of the general systems. The presented method provides an accessible way of obtaining the probability density functions without solving the FokkerPlanck partial differential equations [18] or approximating their solutions.

The rest of the paper is organized as follows. In Section 2, closed-form solution processes of the multidimensional systems are obtained through utilizing the result of A. G. Ladde and G. S. Ladde [19] piecewisely on the intervals between jumps. The problem of finding the closed-form probability density functions is investigated in a systematic and coherent way. In Section 3 the probability density function of the solution process of onedimensional linear homogeneous system of Itô-Doob type stochastic differential equations is derived. This is an extension of the geometric Brownian motion processes [20]. Then, by using the concept of modal matrix, the probability density function of the solution process of $n$-dimensional linear homogeneous systems is obtained in Section 4 . The probability distribution of the solution process of the system with continuous dynamic consisting of only drift part and additive noise, namely, a Ornstein-Uhlenbeck system [18], is extended to hybrid system in Section 5. Some concluding remarks are given in Section 6.

\section{Model Formulation}

In this section, we develop a conceptual stochastic model for dynamic processes in chemical, biological, engineering, medical, physical, and social science $[19,21,22]$ that are under the influence of discrete time events. The continuous time dynamic of the stochastic model between jumps follows a first-order linear non-homogeneous system of Itô-Doob type stochastic differential equations. At random times, governed by a non-homogeneous Poisson process, the coefficients of the continuous time dynamic are switched, and the process is multiplied by a random factor which results in a discontinuous jump.

Let $x(t)$ be a real $n$-dimensional process. $A_{k}$ and $B_{k}^{j}$ are $n \times n$ matrices for any $k \in \mathbb{N} \cup\{0\}$ and $j=1,2, \ldots, q$. Let $C_{k}^{r}$ be $n$-dimensional vectors for any $k \in \mathbb{N} \cup\{0\}$ and $r=1,2, \ldots, p$. Let the continuous dynamic of the process $x(t)$ be determined by the following system of stochastic differential equations:

$$
d x(t)=A_{N(t)} x(t) d t+\sum_{j=1}^{q} B_{N(t)}^{j} x(t) d w_{j}(t)+\sum_{r=1}^{p} C_{N(t)}^{r} d \bar{w}_{r}(t), \quad t \geq t_{0}, x\left(t_{0}\right)=x_{0}>0,
$$


where $w(t)=\left(w_{1}(t), \ldots, w_{q}(t)\right)$ and $\bar{w}(t)=\left(\bar{w}_{1}(t), \ldots, \bar{w}_{p}(t)\right)$ are independent $q$-dimensional and $p$-dimensional standard Wiener processes, and $N(t)$ is a non-homogeneous Poisson process with intensity $\lambda(t)$. Here, we denote $x=\left(x^{1}, x^{2}, \ldots, x^{n}\right)>0$ as $x^{i}>0$ for all $i=1,2, \ldots, n$. When $C_{k}^{r}=0$ for all $k$ and $r$, system (2.1) reduces to a first-order linear homogeneous system of Itô-Doob type stochastic differential equations, given below

$$
d x(t)=A_{N(t)} x(t) d t+\sum_{j=1}^{q} B_{N(t)}^{j} x(t) d w_{j}(t), \quad t \geq t_{0}, x\left(t_{0}\right)=x_{0}>0
$$

Here, $A_{k}$ and $B_{k}^{j}$ are such that solution process $x(t)$ of (2.2) is nonnegative.

To obtain solution process of system (2.1), we first consider the solution process of the initial-value system when $A, B^{j}$, and $C^{r}$ s are fixed over time, that is, the solution process on a subinterval between jumps. Under the condition that the matrices $A, B^{1}, B^{2}, \ldots, B^{q}$ pairwise commute, the solution can be explicitly obtained; see A. G. Ladde and G. S. Ladde [19] or Movellan [23]. We state the result in the following lemma.

Lemma 2.1. Let $x(t) \equiv x\left(t, t_{0}, x_{0}\right)$ be the solution of the following initial value problem (IVP):

$$
d x(t)=A x(t) d t+\sum_{j=1}^{q} B^{j} x(t) d w_{j}(t)+\sum_{r=1}^{p} C^{r} d \bar{w}_{r}(t), \quad t \geq t_{0}, x\left(t_{0}\right)=x_{0},
$$

then the $x(t)$ is expressed by

$$
\begin{aligned}
x(t) \equiv & x\left(t, t_{0}, x_{0}\right)=\exp \left[\left(A-\frac{1}{2} \sum_{j=1}^{q}\left(B^{j}\right)^{2}\right)\left(t-t_{0}\right)+\sum_{j=1}^{q} B^{j}\left(w_{j}(t)-w_{j}\left(t_{0}\right)\right)\right] x_{0} \\
& +\sum_{r=1}^{p} \int_{t_{0}}^{t} \exp \left[\left(A-\frac{1}{2} \sum_{j=1}^{q}\left(B^{j}\right)^{2}\right)(t-s)+\sum_{j=1}^{q} B^{j}\left(w_{j}(t)-w_{j}(s)\right)\right] C^{r} d \bar{w}_{r}(s)
\end{aligned}
$$

provided that $A B^{j}=B^{j} A$ and $B^{j} B^{j^{\prime}}=B^{j^{\prime}} B^{j}$ for all $j, j^{\prime}=1,2, \ldots, q$.

Let $x\left(t, T_{k}, x_{k}\right)$ be the solution to system (2.3) with $t_{0}:=T_{k}, x_{0}:=x_{k}, A:=A_{k}, B^{j}:=$ $B_{k^{\prime}}^{j}$ and $C^{r}:=C_{k}^{r}$. Now, we consider the following system of two interconnected stochastic dynamics:

$$
\begin{gathered}
d x(t)=A_{k-1} x(t) d t+\sum_{k=1}^{q} B_{k-1}^{j} x(t) d w_{j}(t)+\sum_{r=1}^{p} C_{k-1}^{r} d \bar{w}_{r}(t), \quad T_{k-1} \leq t<T_{k}, x\left(T_{k-1}\right)=x_{k-1}, \\
x_{k}=z_{k} x\left(T_{k}^{-}, T_{k-1}, x_{k-1}\right),
\end{gathered}
$$

where $z_{k}, k=1,2,3, \ldots$, are iid positive random variables with $z_{0}=1$, and $x\left(T_{k}^{-}, T_{k-1}, x_{k-1}\right)=$ $\lim _{t \rightarrow T_{k}^{-}} x\left(t, T_{k-1}, x_{k-1}\right)$. Here, we assume that $N(t), w(t), \bar{w}(t)$, and $z_{k}$ are independent. 
By applying Lemma 2.1, piecewisely, on each interval between jumps, we then obtain the solution process of system (2.5). The result is given in the following proposition.

Proposition 2.2. If $A_{k} B_{k}^{j}=B_{k}^{j} A_{k}$ and $B_{k}^{j} B_{k}^{j^{\prime}}=B_{k}^{j^{\prime}} B_{i}^{j}$ for all $k \in \mathbb{N} \cup\{0\}$ and $j, j^{\prime}=1,2, \ldots, q$, then the solution to the system (2.5) is given by

$$
\begin{aligned}
& x(t)=\left(\prod_{k=1}^{N(t)} z_{k}\right) \exp \left[\left(A_{N(t)}-\frac{1}{2} \sum_{j=1}^{q}\left(B_{N(t)}^{j}\right)^{2}\right)\left(t-T_{N(t)}\right)+\sum_{j=1}^{q} B_{N(t)}^{j}\left(w_{j}(t)-w_{j}\left(T_{N(t)}\right)\right)\right] \\
& \times \prod_{k=1}^{N(t)} \exp \left[\left(A_{k-1}-\frac{1}{2} \sum_{j=1}^{q}\left(B_{k-1}^{j}\right)^{2}\right)\left(T_{k}-T_{k-1}\right)+\sum_{j=1}^{q} B_{k-1}^{j}\left(w_{j}\left(T_{k}\right)-w_{j}\left(T_{k-1}\right)\right)\right] x_{0} \\
& +\sum_{i=1}^{N(t)}\left(\prod_{k=i}^{N(t)} z_{k}\right) \exp \left[\left(A_{N(t)}-\frac{1}{2} \sum_{j=1}^{q}\left(B_{N(t)}^{j}\right)^{2}\right)\left(t-T_{N(t)}\right)\right. \\
& \left.+\sum_{j=1}^{q} B_{N(t)}^{j}\left(w_{j}(t)-w_{j}\left(T_{N(t)}\right)\right)\right] \\
& \times \prod_{k=i+1}^{N(t)} \exp \left[\left(A_{k-1}-\frac{1}{2} \sum_{j=1}^{q}\left(B_{k-1}^{j}\right)^{2}\right)\left(T_{k}-T_{k-1}\right)+\sum_{j=1}^{q} B_{k-1}^{j}\left(w_{j}\left(T_{k}\right)-w_{j}\left(T_{k-1}\right)\right)\right] \\
& \times\left[\sum _ { r = 1 } ^ { p } \int _ { T _ { i - 1 } } ^ { T _ { i } } \operatorname { e x p } \left[\left(A_{i-1}-\frac{1}{2} \sum_{j=1}^{q}\left(B_{i-1}^{j}\right)^{2}\right)\left(T_{i}-s\right)\right.\right. \\
& \left.\left.+\sum_{j=1}^{q} B_{i-1}^{j}\left(w_{j}\left(T_{i}\right)-w_{j}(s)\right)\right] C_{i-1}^{r} d \bar{w}_{r}(s)\right] \\
& +\sum_{r=1}^{p} \int_{T_{N(t)}}^{t} \exp \left[\left(A_{N(t)}-\frac{1}{2} \sum_{j=1}^{q}\left(B_{N(t)}^{j}\right)^{2}\right)(t-s)+\sum_{j=1}^{q} B_{N(t)}^{j}\left(w_{j}(t)-w_{j}(s)\right)\right] C_{N(t)}^{r} d \bar{w}_{r}(s) \text {. }
\end{aligned}
$$

Proof. By applying the result of (2.4) on the subintervals $\left[T_{k-1}, T_{k}\right)$, for $k=1, \ldots, N(t)$, and $\left[T_{N(t)}, t\right)$, we have the solution to system $(2.5)$ as the following piecewise function:

$$
x(s)= \begin{cases}x\left(s, t_{0}, x_{0}\right) & x\left(t_{0}\right)=x_{0}, \quad t_{0} \leq s<T_{1}, \\ x\left(s, T_{1}, x_{1}\right) & x\left(T_{1}\right)=x_{1}, \quad T_{1} \leq s<T_{2}, \\ \cdots & \\ x\left(s, T_{i}, x_{i}\right) & x\left(T_{i}\right)=x_{k}, \quad T_{k} \leq s<T_{k+1}, \\ \cdots & \\ x\left(s, T_{N(t)}, x_{N(t)}\right) & x\left(T_{N(t)}\right)=x_{N(t)}, \quad T_{N(t)} \leq s<t,\end{cases}
$$


where $x\left(s, T_{k}, x_{k}\right)$ is a solution process in (2.4) with $t_{0}:=T_{k}, x_{0}:=x_{k}, A:=A_{k}, B^{j}:=B_{k}^{j}$, and $C^{r}:=C_{k}^{r}$, then, we have

$$
\begin{aligned}
& x(t) \\
& =x\left(t, T_{N(t)}, x_{N(t)}\right) \\
& =\exp \left[\left(A_{N(t)}-\frac{1}{2} \sum_{j=1}^{q}\left(B_{N(t)}^{j}\right)^{2}\right)\left(t-T_{N(t)}\right)+\sum_{j=1}^{q} B_{N(t)}^{j}\left(w_{j}(t)-w_{j}\left(T_{N(t)}\right)\right)\right] x_{N(t)} \\
& +\sum_{r=1}^{p} \int_{T_{N(t)}}^{t} \exp \left[\left(A_{N(t)}-\frac{1}{2} \sum_{j=1}^{q}\left(B_{N(t)}^{j}\right)^{2}\right)(t-s)+\sum_{j=1}^{q} B_{N(t)}^{j}\left(w_{j}(t)-w_{j}(s)\right)\right] C_{N(t)}^{r} d \bar{w}_{r}(s) \\
& =z_{N(t)} \exp \left[\left(A_{N(t)}-\frac{1}{2} \sum_{j=1}^{q}\left(B_{N(t)}^{j}\right)^{2}\right)\left(t-T_{N(t)}\right)+\sum_{j=1}^{q} B_{N(t)}^{j}\left(w_{j}(t)-w_{j}\left(T_{N(t)}\right)\right)\right] \\
& \times\left[\operatorname { e x p } \left[\left(A_{N(t)-1}-\frac{1}{2} \sum_{j=1}^{q}\left(B_{N(t)-1}^{j}\right)^{2}\right)\left(T_{N(t)}-T_{N(t)-1}\right)\right.\right. \\
& \left.+\sum_{j=1}^{q} B_{N(t)-1}^{j}\left(w_{j}\left(T_{N(t)}\right)-w_{j}\left(T_{N(t)-1}\right)\right)\right] x_{N(t)-1} \\
& +\sum_{r=1}^{p} \int_{T_{N(t)-1}}^{T_{N(t)}}\left[\left(A_{N(t)-1}-\frac{1}{2} \sum_{j=1}^{q}\left(B_{N(t)-1}^{j}\right)^{2}\right)\left(T_{N(t)}-s\right)\right. \\
& +\sum_{r=1}^{p} \int_{T_{N(t)}^{t}} \exp \left[\left(\sum_{j=1}^{q} B_{N(t)-1}^{j}\left(w_{j}\left(T_{N(t)}\right)-w_{j}(s)\right)\right] C_{N(t)-1}^{r} d \bar{w}_{r}(s)\right] \\
& \left.\left.A_{N(t)}-\frac{1}{2} \sum_{j=1}^{q}\left(B_{N(t)}^{j}\right)^{2}\right)(t-s)+\sum_{j=1}^{q} B_{N(t)}^{j}\left(w_{j}(t)-w_{j}(s)\right)\right] C_{N(t)}^{r} d w_{r}(s) .
\end{aligned}
$$

Next, substitute $x_{N(t)-1}=z_{N(t)-1} x\left(T_{N(t)-1}^{-}, T_{N(t)-2}, x_{N(t)-2}\right)=z_{N(t)-1} x\left(T_{N(t)-1}, T_{N(t)-2}, x_{N(t)-2}\right)$. The term $x\left(T_{N(t)-1}^{-}, T_{N(t)-2}, x_{N(t)-2}\right)$ can be replaced by $x\left(T_{N(t)-1}, T_{N(t)-2}, x_{N(t)-2}\right)$ because the solution process is continuous between jumps. Repeating the substitution gives the desired result.

In the following, we present two important particular by-products of Proposition 2.2. When $C_{k}^{r}=0$ for all $k$ and $r$, system (2.5) reduces to the following first-order linear homogeneous system of Itô-Doob type stochastic differential equations with jumps:

$$
\begin{gathered}
d x(t)=A_{k-1} x(t) d t+\sum_{k=1}^{q} B_{k-1}^{j} x(t) d w_{j}(t), \quad T_{k-1} \leq t<T_{k}, x\left(T_{k-1}\right)=x_{k-1}, \\
x_{k}=z_{k} x\left(T_{k}^{-}, T_{k-1}, x_{k-1}\right) .
\end{gathered}
$$


The solution of the above system is given in the following corollary. The result follows from Proposition 2.2 by letting $C_{k}^{r}$ be zero for all $k$ and $r$.

Corollary 2.3. If $A_{k} B_{k}^{j}=B_{k}^{j} A_{k}$ and $B_{k}^{j} B_{k}^{j^{\prime}}=B_{k}^{j^{\prime}} B_{i}^{j}$ for all $k \in \mathbb{N} \cup\{0\}$ and $j, j^{\prime}=1,2, \ldots, q$, then the solution of system (2.9) is given by

$$
\begin{aligned}
x(t)= & \left(\prod_{k=1}^{N(t)} z_{k}\right) \exp \left[\left(A_{N(t)}-\frac{1}{2} \sum_{j=1}^{q}\left(B_{N(t)}^{j}\right)^{2}\right)\left(t-T_{N(t)}\right)+\sum_{j=1}^{q} B_{N(t)}^{j}\left(w_{j}(t)-w_{j}\left(T_{N(t)}\right)\right)\right] \\
& \times \prod_{k=1}^{N(t)} \exp \left[\left(A_{k-1}-\frac{1}{2} \sum_{j=1}^{q}\left(B_{k-1}^{j}\right)^{2}\right)\left(T_{k}-T_{k-1}\right)+\sum_{j=1}^{q} B_{k-1}^{j}\left(w_{j}\left(T_{k}\right)-w_{j}\left(T_{k-1}\right)\right)\right] x_{0} .
\end{aligned}
$$

In the case when $B_{k}^{j}$ are zeros for all $j$ and $k$, system (2.5) becomes a linear system with additive noise. The continuous dynamics between jumps are now governed by OrnsteinUhlenbeck equations as follows:

$$
\begin{gathered}
d x(t)=A_{k-1} x(t) d t+\sum_{r=1}^{p} C_{k-1}^{r} d \bar{w}_{r}(t), \quad T_{k-1} \leq t<T_{k}, x\left(T_{k-1}\right)=x_{k-1} \\
x_{k}=z_{k} x\left(T_{k}^{-}, T_{k-1}, x_{k-1}\right) .
\end{gathered}
$$

Denote $C_{k}=\left(C_{k^{\prime}}^{1}, C_{k^{\prime}}^{2}, \ldots, C_{k}^{p}\right)$ for all $k$, then the above system can be rewritten as

$$
\begin{gathered}
d x(t)=A_{k-1} x(t) d t+C_{k-1} d \bar{w}(t), \quad T_{k-1} \leq t<T_{k}, x\left(T_{k-1}\right)=x_{k-1}, \\
x_{k}=z_{k} x\left(T_{k}^{-}, T_{k-1}, x_{k-1}\right),
\end{gathered}
$$

where $C_{k}$ 's are $n \times p$ matrices, and $\bar{w}(t)$ is a $p$-dimensional standard Wiener process.

Corollary 2.4. The solution of system (2.11) is given by

$$
\begin{aligned}
x(t)= & \left(\prod_{k=1}^{N(t)} z_{k}\right) \exp \left[A_{N(t)}\left(t-T_{N(t)}\right)\right] \prod_{k=1}^{N(t)} \exp \left[A_{k-1}\left(T_{k}-T_{k-1}\right)\right] x_{0} \\
& +\sum_{i=1}^{N(t)}\left(\prod_{k=i}^{N(t)} z_{k}\right) \exp \left[A_{N(t)}\left(t-T_{N(t)}\right)\right] \prod_{k=i+1}^{N(t)} \exp \left[A_{k-1}\left(T_{k}-T_{k-1}\right)\right] \\
& \times\left[\sum_{r=1}^{p} \int_{T_{i-1}}^{T_{i}} \exp \left[A_{i-1}\left(T_{i}-s\right)\right] C_{i-1}^{r} d \bar{w}_{r}(s)\right] \\
& +\sum_{r=1}^{p} \int_{T_{N(t)}^{t}}^{t} \exp \left[A_{N(t)}(t-s)\right] C_{N(t)}^{r} d \bar{w}_{r}(s) .
\end{aligned}
$$




\section{Probability Distribution of One-Dimensional Linear Homogeneous Models}

In this section, we will derive the probability density function of the solution process of the scalar version of system (2.9). Now, $x(t)$ takes values in $\mathbb{R}_{+}$, and in this case, $A_{k}$ and $B_{k}$ are scalars for all $k$ in the system (2.9) and the solution process (2.10). Some auxiliary results are presented below. The following lemma provides the joint density function of the jump times given the number of jumps due to the non-homogeneous Poisson process $N(t)$. The proof of this result can be found in many textbooks, for example, see Cox and Lewis [24] or Crowder et al. [25].

Lemma 3.1. For a non-homogeneous Poisson process $N(t)$ with intensity $\lambda(t)$, the joint density function of the jump times $T_{1}, T_{2}, \ldots, T_{l}$ conditioned on $N(t)=l$ is given by

$$
f_{T_{1}, T_{2}, \ldots, T_{l} \mid N(t)=l}\left(t_{1}, t_{2}, \ldots, t_{l}\right)=\frac{l ! \prod_{k=1}^{l} \lambda\left(t_{k}\right)}{\left(\int_{0}^{t} \lambda(u) d u\right)^{l}}
$$

Next lemma gives the conditional probability density function of $x(t)$ given the number of jumps and the jump times.

Lemma 3.2. Given that $N(t)=l$, and $T_{1}=t_{1}, \ldots, T_{l}=t_{l}, x(t)$ has a probability density function as

$$
f_{x(t) \mid N(t)=l, t_{1}, \ldots, t_{l}}(x)=\frac{1}{x} \int_{-\infty}^{\infty} h^{* l}(\ln x-s) \phi(s ; \mu, \sigma) d s, \quad x>0,
$$

where $h^{* l}$ is the lth convolution of the common probability density function $h$ of $\ln z_{k}$, for $k=$ $1,2, \ldots, l$, and $\phi(\cdot ; \mu, \sigma)$ denotes the normal density function with mean $\mu$ and variance $\sigma$.

Proof. Given that $N(t)=l$, and $T_{1}=t_{1}, \ldots, T_{l}=t_{l}$, from (2.10), we have

$$
\begin{aligned}
\ln x(t)= & \sum_{k=1}^{l} \ln z_{k}+\ln x_{0}+\left[\left(A_{l}-\frac{B_{l}^{2}}{2}\right)\left(t-t_{l}\right)+B_{l}\left(w(t)-w\left(t_{l}\right)\right)\right] \\
& +\sum_{k=1}^{l}\left[\left(A_{k-1}-\frac{B_{k-1}^{2}}{2}\right)\left(t_{k}-t_{k-1}\right)+B_{k-1}\left(w\left(t_{k}\right)-w\left(t_{k-1}\right)\right)\right] \\
= & V+S,
\end{aligned}
$$

where we denote $V=\sum_{k=1}^{l} \ln z_{k}$ and $S$ as the sum of last three terms in (3.3).

Since $h$ is the common probability density function of $\ln z_{k}, V$ as the sum of $l$ iid random variables has the probability density function as the $l$ th convolution $h^{* l}(v)$. We further note that $S$ is the sum of $l+1$ independent normal variables due to the independent 
increment property of Wiener process. Then $S$ is normally distributed with mean and variance as

$$
\begin{gathered}
\mu \equiv \mu\left(t, t_{1}, \ldots, t_{l}\right)=\ln x_{0}+\left(A_{l}-\frac{B_{l}^{2}}{2}\right)\left(t-t_{l}\right)+\sum_{k=1}^{l}\left(A_{k-1}-\frac{B_{k-1}^{2}}{2}\right)\left(t_{k}-t_{k-1}\right), \\
\sigma \equiv \sigma\left(t, t_{1}, \ldots, t_{l}\right)=B_{l}^{2}\left(t-t_{l}\right)+\sum_{k=1}^{l} B_{k-1}^{2}\left(t_{k}-t_{k-1}\right) .
\end{gathered}
$$

Since $z_{k}$ and $w(t)$ are independent, then $V$ and $S$ are also independent. Using transformation method [26] on $\ln x(t)=V+S$, we can obtain the conditional probability density function of $\ln x(t)$ as

$$
f_{\ln x(t) \mid N(t)=l, t_{1}, \ldots, t_{l}}(x)=\int_{-\infty}^{\infty} h^{* l}(x-s) \phi(s ; \mu, \sigma) d s .
$$

If follows that

$$
\begin{aligned}
f_{x(t) \mid N(t)=l, t_{1}, \ldots, t_{l}}(x) & =f_{\ln x(t) \mid N(t)=l, t_{1}, \ldots, t_{l}}(\ln x) \frac{1}{x} \\
& =\frac{1}{x} \int_{-\infty}^{\infty} h^{* l}(\ln x-s) \phi(s ; \mu, \sigma) d s, \quad x>0 .
\end{aligned}
$$

Having obtained the conditional probability density function of $x(t)$, we can derive the marginal probability distribution of the solution process in the one-dimensional case as follows.

Proposition 3.3. The probability density function of the scalar version of the solution process $x(t)$ to the system (2.5) is given by

$$
\begin{aligned}
f_{x(t)}(x)=\sum_{l=0}^{\infty}[ & {\left[\int_{0}^{t} \cdots \int_{0}^{t_{3}} \int_{0}^{t_{2}} \int_{-\infty}^{\infty} h^{* l}(\ln x-s) \phi(s ; \mu, \sigma) d s \prod_{k=1}^{l} \lambda\left(t_{k}\right) d t_{1} d t_{2} \cdots d t_{l}\right.} \\
& \left.\times \frac{1}{x} \exp \left[-\int_{0}^{t} \lambda(u) d u\right]\right] .
\end{aligned}
$$

Proof. From (3.1) and (3.2), the joint density function of $x(t), T_{1}, \ldots, T_{l}$ given the condition $N(t)=l$ is given by

$$
\begin{aligned}
f_{x(t), T_{1}, \ldots, T_{l} \mid N(t)=l}\left(x, t_{1}, \ldots, t_{l}\right) & =f_{x(t) \mid N(t)=l, t_{1}, \ldots, t_{l}}(x) f_{T_{1}, T_{2}, \ldots, T_{l} \mid N(t)=l}\left(t_{1}, t_{2}, \ldots, t_{l}\right) \\
& =\frac{1}{x} \int_{-\infty}^{\infty} h^{* l}(\ln x-s) \phi(s ; \mu, \sigma) d s \frac{l ! \prod_{k=1}^{l} \lambda\left(t_{k}\right)}{\left(\int_{0}^{t} \lambda(u) d u\right)^{l}}
\end{aligned}
$$


Integrating with respect to $t_{1}, t_{2}, \ldots$, and $t_{l}$ gives the probability density function of $x(t)$ given that $N(t)=l$ as

$$
f_{x(t) \mid N(t)=l}(x)=\int_{0}^{t} \cdots \int_{0}^{t_{3}} \int_{0}^{t_{2}} \int_{-\infty}^{\infty} h^{* l}(\ln x-s) \phi(s ; \mu, \sigma) d s \prod_{k=1}^{l} \lambda\left(t_{k}\right) d t_{1} d t_{2} \cdots d t_{l} \frac{l !}{x\left(\int_{0}^{t} \lambda(u) d u\right)^{l}}
$$

By multiplying the above density by the probability of $l$ jumps, namely,

$$
\operatorname{Pr}(N(t)=l)=\exp \left[-\int_{0}^{t} \lambda(u) d u\right] \frac{\left(\int_{0}^{t} \lambda(u) d u\right)^{l}}{l !}
$$

and then taking the summation over $l$, the marginal probability density function of $x(t)$ given in (3.7) is established.

\section{Probability Distribution of Multivariate Linear Homogeneous Models}

In this section, we will derive the probability density function of the solution process $x(t)$ for the $n$-dimensional stochastic system (2.9) under the following assumptions.

(i) The drift and diffusion coefficients, $A_{k}$ and $B_{k}^{j}$ for all $k \in \mathbb{N} \cup\{0\}$ and $j=1,2, \ldots, q$, are diagonalizable.

(ii) The coefficients in each regime pairwise commute, that is, $A_{k} B_{k}^{j}=B_{k}^{j} A_{k}$ and $B_{k}^{j} B_{k}^{l}=$ $B_{k}^{l} B_{k}^{j}$ for all $k \in \mathbb{N} \cup\{0\}$ and $j, l=1,2, \ldots, q$.

(iii) $A_{k} \in \mathcal{C}$ for $k \in \mathbb{N} \cup\{0\}$, where $\mathcal{C}$ denotes the set of $n \times n$ diagonalizable matrices whose eigenvector matrix, $M$, is such that $\mathrm{M}^{-1} x>0$ for any $x>0$.

We first consider the stochastic system on the interval between jumps. Given that $N(t)=l$ and $T_{1}=t_{1}, T_{2}=t_{2}, \ldots, T_{l}=t_{l}$, consider the following SDE on $\left[t_{k}, t_{k+1}\right)$, for some $k=0,1, \ldots, l$ :

$$
d x(s)=A_{k} x(s) d t+\sum_{j=1}^{q} B_{k}^{j} x(s) d w_{j}(s), \quad t_{k} \leq s<t_{k+1}, x\left(t_{k}\right)=x_{k} .
$$

In the following, we provide the necessary background material that will be used, subsequently. The following result provides a way to find a modal matrix that can diagonalize the coefficients in the above system.

Theorem 4.1 (see [27]). A set of diagonalizable matrices commutes if and only if the set is simultaneously diagonalizable, that is, there exists an invertible matrix that can diagonalize all the matrices simultaneously. 
Remark 4.2. In fact, the set of diagonalizable and commuting matrices shares the same set of independent eigenvectors. The eigenvector matrix is the one that simultaneously diagonalizes all the matrices in this set, and the resulting diagonal elements are the eigenvalues of each matrix.

We recall [26] that a random vector $x$ is said to have a $n$-dimensional multivariate normal distribution with mean and covariance matrix, $\mu_{x}$ and $\Sigma_{x}$, if its probability density function is given by

$$
f(x)=(2 \pi)^{-n / 2}\left|\Sigma_{x}\right|^{-1 / 2} \exp \left[-\frac{1}{2}\left(x-\mu_{x}\right)^{T} \Sigma_{x}^{-1}\left(x-\mu_{x}\right)\right] .
$$

The following lemma gives the useful fact that the linear transformation of a multivariate normal random vector is again multivariate normally distributed.

Theorem 4.3 (see [26]). If $x$ has a multivariate normal distribution with mean and covariance matrix, $\mu_{x}$ and $\Sigma_{x}$, then $y=P x+c$ as a linear transformation of $x$ follows also multivariate normal distribution with mean and covariance matrix, $\mu_{y}=P \mu_{x}+c$ and $\Sigma_{y}=P \Sigma_{x} P^{T}$.

Proof. Since $y_{i}=\sum_{j=1}^{n} P_{i j} x_{j}+c_{i}$ and every linear combination of normal random variables is still normal, then $y$ follows a multivariate normal distribution. By the linearity of expectation we have

$$
\begin{gathered}
\mu_{y}=E[y]=E\left[P \mu_{x}+c\right]=P E[x]+c=P \mu_{x}+c \\
\Sigma_{y}=E\left[\left(y-\mu_{y}\right)\left(y-\mu_{y}\right)^{T}\right]=E\left[\left[P\left(x-\mu_{x}\right)\right]\left[P\left(x-\mu_{x}\right)\right]^{T}\right] \\
=E\left[P\left(x-\mu_{x}\right)\left(x-\mu_{x}\right)^{T} P^{T}\right]=P E\left[\left(x-\mu_{x}\right)\left(x-\mu_{x}\right)^{T}\right] P^{T}=P \Sigma_{x} P^{T} .
\end{gathered}
$$

To find the probability density function of $x(s)$ satisfying the SDE (4.1), we need to introduce some notations and definitions that will be used, subsequently. First note that according to Theorem 4.1 and Remark $4.2, A_{k} \mathrm{~s}$ and $B_{k}^{j} \mathrm{~s}$ have the same eigenvector matrix, denoted by $M_{k}$. Moreover, $\tilde{A}_{k} \equiv M_{k}^{-1} A_{k} M_{k}=\operatorname{diag}\left(\tilde{a}_{k}^{1}, \ldots, \tilde{a}_{k}^{n}\right)$ and $\tilde{B}_{k}^{j} \equiv M_{k}^{-1} B_{k}^{j} M_{k}=$ $\operatorname{diag}\left(\tilde{b}_{k, j}^{1}, \ldots, \tilde{b}_{k, j}^{n}\right)$ where $\left\{\tilde{a}_{k}^{1}, \ldots, \tilde{a}_{k}^{n}\right\}$ and $\left\{\tilde{b}_{k, j}^{1}, \ldots, \tilde{b}_{k, j}^{n}\right\}$ are the sets of eigenvalues of $A_{k}$ and $B_{k^{\prime}}^{j}$ respectively, for all $k, j$. Next, we define a linear transformation $y=M_{k}^{-1} x$, then $x=M_{k} y$. Define $\ln y=\left(\ln y^{1}, \ln y^{2}, \ldots, \ln y^{n}\right)$ [28].

The below proposition gives the probability distribution of solution process $x(t)$ on the interval $\left[t_{k}, t_{k+1}\right)$ over which the coefficients are constant.

Lemma 4.4. Under assumptions (i)-(iii), the process $x(s)$ satisfying the SDE (4.1) has a probability density function given by

$$
\begin{aligned}
f_{x(s)}(x)= & (2 \pi)^{-n / 2}\left|\Sigma_{k}(s)\right|^{-1 / 2}\left|M_{k}^{-1}\right| \prod_{i=1}^{n} \frac{1}{\left(M_{k}^{-1} x\right)^{i}} \\
& \times \exp \left[-\frac{1}{2}\left(\ln \left(M_{k}^{-1} x\right)-\mu_{k}(s)\right)^{T}\left(\Sigma_{k}(s)\right)^{-1}\left(\ln \left(M_{k}^{-1} x\right)-\mu_{k}(s)\right)\right], \quad \text { for } s \in\left[t_{k}, t_{k+1}\right),
\end{aligned}
$$


where

$$
\mu_{k}(s)=\left(\begin{array}{c}
\ln y^{1}\left(t_{k}\right)+\left(\tilde{a}_{k}^{1}-\frac{1}{2} \sum_{j=1}^{q}\left(\tilde{b}_{k, j}^{1}\right)^{2}\right)\left(s-t_{k}\right) \\
\ln y^{2}\left(t_{k}\right)+\left(\tilde{a}_{k}^{2}-\frac{1}{2} \sum_{j=1}^{q}\left(\tilde{b}_{k, j}^{2}\right)^{2}\right)\left(s-t_{k}\right) \\
\vdots \\
\ln y^{n}\left(t_{k}\right)+\left(\tilde{a}_{k}^{n}-\frac{1}{2} \sum_{j=1}^{q}\left(\tilde{b}_{k, j}^{n}\right)^{2}\right)\left(s-t_{k}\right)
\end{array}\right), \quad B_{k}^{*}=\left(\begin{array}{cccc}
\tilde{b}_{k, 1}^{1} & \tilde{b}_{k, 2}^{1} & \cdots & \tilde{b}_{k, q}^{1} \\
\tilde{b}_{k, 1}^{2} & \tilde{b}_{k, 2}^{2} & \cdots & \tilde{b}_{k, q}^{2} \\
\vdots & \vdots & \vdots & \vdots \\
\tilde{b}_{k, 1}^{n} & \tilde{b}_{k, 2}^{n} & \cdots & \tilde{b}_{k, q}^{n}
\end{array}\right),
$$

Proof. For $s \in\left[t_{k}, t_{k+1}\right)$, we have $y(s)=M_{k}^{-1} x(s)$, and $x(s)=M_{k} y(s)$. By multiplying $M_{k}^{-1}$ on both sides of the SDE (4.1), we obtain the transformed SDE as follows:

$$
\begin{aligned}
& M_{k}^{-1} d x(s)=M_{k}^{-1} A_{k} x(s) d s+\sum_{j=1}^{q} M_{k}^{-1} B_{k}^{j} x(s) d w_{j}(s) \\
& \Longrightarrow d M_{k}^{-1} x(s)=M_{k}^{-1} A_{k} M_{k} y(s) d s+\sum_{j=1}^{q} M_{k}^{-1} B_{k}^{j} M_{k} y(s) d w_{j}(s) \\
& \Longrightarrow d y(s)=\tilde{A}_{k} y(s) d s+\sum_{j=1}^{q} \tilde{B}_{k}^{j} y(s) d w_{j}(s)
\end{aligned}
$$

where $\widetilde{A}_{k}$ and $\widetilde{B}_{k}^{j}$ are diagonal matrices as defined before.

From the application of Lemma 2.1 with $C_{k}^{r}=0$, the solution process of the transformed system (4.6) is

$$
y(s)=\exp \left[\left(\tilde{A}-\frac{1}{2} \sum_{j=1}^{q}\left(\tilde{B}_{k}^{j}\right)^{2}\right)\left(s-t_{k}\right)+\sum_{j=1}^{q} \tilde{B}_{k}^{j}\left(w_{j}(s)-w_{j}\left(t_{k}\right)\right)\right] y_{k}
$$

for $s \in\left[t_{k}, t_{k+1}\right)$.

It follows from assumption (iii) that $y(s)=M_{k}^{-1} x(s)>0$ since $x(s)>0$, then, we can rewrite system (4.7) in the following form:

$$
\ln y(s)=\mu_{k}(s)+B_{k}^{*}\left(w(s)-w\left(t_{k}\right)\right)
$$

where $\mu_{k}(s)$ and $B_{k}^{*}$ are defined above. 
Since $w(t)$ is a standard Wiener process, then $w(s)-w\left(t_{k}\right)$ has a multivariate normal distribution with mean zero and covariance matrix $\left(s-t_{k}\right) I_{n}$, where $I_{n}$ is the $n \times n$ identity matrix. Then, by Theorem 4.3, $\ln y(s)$ as a linear transformation of $w(s)-w\left(t_{k}\right)$ is also multivariate normally distributed with mean $\mu_{k}(s)$ and covariance matrix $\Sigma_{k}(s)$, where

$$
\Sigma_{k}(s)=B_{k}^{*}\left[\left(s-t_{k}\right) I_{n}\right]\left(B_{k}^{*}\right)^{T}=\left(s-t_{k}\right) B_{k}^{*}\left(B_{k}^{*}\right)^{T},
$$

and the $(u, v)$ th element of $\Sigma_{k}(s)$ is $\left(s-t_{k}\right) \sum_{j=1}^{q} \tilde{b}_{k, j}^{u} \tilde{b}_{k, j^{\prime}}^{v}$ for $u, v=1,2, \ldots, n$. The probability density function of $\ln y(s)$ is

$$
f_{\ln y(s)}(\tilde{y})=(2 \pi)^{-n / 2}\left|\Sigma_{k}(s)\right|^{-1 / 2} \exp \left[-\frac{1}{2}\left(\tilde{y}-\mu_{k}(s)\right)^{T}\left(\Sigma_{k}(s)\right)^{-1}\left(\tilde{y}-\mu_{k}(s)\right)\right] .
$$

We now apply the method of transformation from $y_{\ln }(s)$ to $y(s)$, then,

$$
f_{y(s)}(y)=f_{\ln y(s)}\left(y_{\ln }\right)\left|\operatorname{det}\left(J_{1}\right)\right|
$$

where $\ln y=\left(\ln y^{1}, \ln y^{2}, \ldots, \ln y^{n}\right)$, and $J_{1}$ is the Jacobian matrix. The Jacobian determinant can be computed as

$$
\operatorname{det}\left(J_{1}\right)=\left|\begin{array}{cccc}
\frac{\partial \ln y^{1}}{\partial y^{1}} & \frac{\partial \ln y^{1}}{\partial y^{2}} & \cdots & \frac{\partial \ln y^{1}}{\partial y^{n}} \\
\frac{\partial \ln y^{2}}{\partial y^{1}} & \frac{\partial \ln y^{2}}{\partial y^{2}} & \cdots & \frac{\partial \ln y^{2}}{\partial y^{n}} \\
\vdots & \vdots & \vdots & \vdots \\
\frac{\partial \ln y^{n}}{\partial y^{1}} & \frac{\partial \ln y^{n}}{\partial y^{2}} & \cdots & \frac{\partial \ln y^{n}}{\partial y^{n}}
\end{array}\right|=\left|\begin{array}{ccccc}
\frac{1}{y^{1}} & 0 & \cdots & 0 \\
0 & \frac{1}{y^{2}} & \cdots & 0 \\
\vdots & \vdots & \ddots & \vdots \\
0 & 0 & \cdots & \frac{1}{y^{n}}
\end{array}\right|=\prod_{i=1}^{n} \frac{1}{y^{i}}
$$

Then, from (4.10) and (4.11), we have the probability density function of $y(s)$ as

$$
\begin{aligned}
f_{y(s)}(y)= & (2 \pi)^{-n / 2}\left|\Sigma_{k}(s)\right|^{-1 / 2} \prod_{i=1}^{n} \frac{1}{y^{i}} \\
& \times \exp \left[-\frac{1}{2}\left(\ln y-\mu_{k}(s)\right)^{T}\left(\Sigma_{k}(s)\right)^{-1}\left(\ln y-\mu_{k}(s)\right)\right] .
\end{aligned}
$$

Next step is to find the probability density function of $x(s)$ by using method of transformation from $y(s)$ to $x(s)$. Since $x(s)=M_{k} y(s)$, or $y(s)=M_{k}^{-1} x(s)$, we have

$$
f_{x(s)}(x)=f_{y(s)}\left(M_{k}^{-1} x\right)\left|\operatorname{det}\left(J_{2}\right)\right|
$$


where

$$
\operatorname{det}\left(J_{2}\right)=\operatorname{det}\left(\frac{\partial}{\partial x^{v}} \sum_{j=1}^{n}\left(M_{k}^{-1}\right)^{u j} x^{j}\right)_{u, v}=\operatorname{det}\left(\left(M_{k}^{-1}\right)^{u v}\right)_{u, v}=\operatorname{det}\left(M_{k}^{-1}\right)
$$

The result follows from combining (4.13) and (4.14).

Now, we are to develop the conditional probability density function of solution process $x(t)$, which is a result parallel to the one-dimensional case in Lemma 3.2.

Lemma 4.5. Under assumptions (i)-(iii) and given that $N(t)=l$, and $T_{1}=t_{1}, T_{2}=t_{2}, \ldots, T_{l}=t_{l}$, the solution process $x(t)$ has a conditional probability density function as

$$
\begin{aligned}
& f_{x(t) \mid N(t)=l, t_{1}, \ldots, t_{l}}(x) \\
& =\int_{\mathbb{R}_{+}^{n}} \ldots \int_{\mathbb{R}_{+}^{n}} f_{x(t), x\left(t_{l}\right), \ldots, x\left(t_{1}\right) \mid N(t)=l, t_{1}, \ldots, t_{l}}\left(x, x_{l}, \ldots, x_{1}\right) d x_{l} \cdots d x_{1} \\
& =\int_{\mathbb{R}_{+}^{n}} \ldots \int_{\mathbb{R}_{+}^{n}}(2 \pi)^{-n / 2}\left|\Sigma_{l}(t)\right|^{-1 / 2}\left|M_{l}^{-1}\right| \prod_{i=1}^{n} \frac{1}{\left(M_{l}^{-1} x\right)^{i}} \\
& \quad \times \exp \left[-\frac{1}{2}\left(\ln \left(M_{l}^{-1} x\right)-\mu_{l}(t)\right)^{T}\left(\Sigma_{l}(t)\right)^{-1}\left(\ln \left(M_{l}^{-1} x\right)-\mu_{l}(t)\right)\right] \\
& \quad \times \prod_{k=0}^{l-1}\left[\int_{0}^{\infty}(2 \pi)^{-n / 2}\left|\Sigma_{k}\left(t_{k+1}\right)\right|^{-1 / 2}\left|M_{k}^{-1}\right| \prod_{i=1}^{n} \frac{1}{\left(M_{k}^{-1}\left(x_{k+1} / z_{k+1}\right)\right)^{i}}\right. \\
& \quad \times \exp \left[-\frac{1}{2}\left(\ln \left(M_{k}^{-1}\left(\frac{x_{k+1}}{z_{k+1}}\right)\right)-\mu_{k}\left(t_{k+1}\right)\right)^{T}\left(\Sigma_{k}\left(t_{k+1}\right)\right)^{-1}\right. \\
& \left.\left.\quad \times\left(\ln \left(M_{k}^{-1}\left(\frac{x_{k+1}}{z_{k+1}}\right)\right)-\mu_{k}\left(t_{k+1}\right)\right)\right] g\left(z_{k+1}\right) \frac{1}{z_{k+1}^{n}} d z_{k+1}\right] d x_{l} \cdots d x_{1},
\end{aligned}
$$

where $g$ is the common probability density function of $z_{k}, k=1,2, \ldots, l$.

Proof. We will apply the result in Lemma 4.4 piecewisely to the system (2.9) under the conditions $N(t)=l$ and $T_{1}=t_{1}, T_{2}=t_{2}, \ldots, T_{l}=t_{l}$. First, we note that the joint probability density function of $\left(x(t), x\left(t_{l}\right), \ldots, x\left(t_{1}\right)\right)$ can be expressed as

$$
\begin{aligned}
& f_{x(t), x\left(t_{l}\right), \ldots, x\left(t_{1}\right) \mid N(t)=l, t_{1}, \ldots, t_{l}}\left(x, x_{l}, \ldots, x_{1}\right) \\
& \quad=f_{x(t) \mid x\left(t_{l}\right), N(t)=l, t_{1}, \ldots, t_{l}}(x) f_{x\left(t_{l}\right) \mid x\left(t_{l-1}\right), N(t)=l, t_{1}, \ldots, t_{l}}\left(x_{l}\right) \cdots f_{x\left(t_{1}\right) \mid N(t)=l, t_{1}, \ldots, t_{l}}\left(x_{1}\right),
\end{aligned}
$$


then, for $k=0,1, \ldots, l-1$, consider that $x\left(t_{k+1}\right)=x\left(t_{k+1}^{-}\right) z_{k+1}$ as a product of two random variables where the first one has the probability density function given in (4.4), and $z_{k+1}$ is the random jump factor at time $t_{k+1}$. By the independence of $x\left(t_{k+1}^{-}\right)$and $z_{k+1}$, we then have

$$
\begin{aligned}
& f_{x\left(t_{k+1}\right) \mid x\left(t_{k}\right), N(t)=l, t_{1}, \ldots, t_{l}}\left(x_{k+1}\right) \\
& =\int_{0}^{\infty} f_{x\left(t_{k+1}^{-}\right) \mid x\left(t_{k}\right), N(t)=l, t_{1}, \ldots, t_{l}}\left(\frac{x_{k+1}}{z_{k+1}}\right) g\left(z_{k+1}\right) \frac{1}{z_{k+1}^{n}} d z_{k+1} \\
& =\int_{0}^{\infty}(2 \pi)^{-n / 2}\left|\Sigma_{k}\left(t_{k+1}\right)\right|^{-1 / 2}\left|M_{k}^{-1}\right| \prod_{i=1}^{n} \frac{1}{\left(M_{k}^{-1}\left(x_{k+1} / z_{k+1}\right)\right)^{i}} \\
& \quad \times \exp \left[-\frac{1}{2}\left(\ln \left(M_{k}^{-1}\left(\frac{x_{k+1}}{z_{k+1}}\right)\right)-\mu_{k}\left(t_{k+1}\right)\right)^{T}\left(\Sigma_{k}\left(t_{k+1}\right)\right)^{-1}\right. \\
& \left.\quad \times\left(\ln \left(M_{k}^{-1}\left(\frac{x_{k+1}}{z_{k+1}}\right)\right)\right)-\mu_{k}\left(t_{k+1}\right)\right] g\left(z_{k+1}\right) \frac{1}{z_{k+1}^{n}} d z_{k+1},
\end{aligned}
$$

then (4.17) can be written as

$$
\begin{aligned}
& f_{x(t), x\left(t_{l}\right), \ldots, x\left(t_{1}\right) \mid N(t)=l, t_{1}, \ldots, t_{l}}\left(x, x_{l}, \ldots, x_{1}\right) \\
&=(2 \pi)^{-n / 2}\left|\Sigma_{l}(t)\right|^{-1 / 2}\left|M_{l}^{-1}\right| \prod_{i=1}^{n} \frac{1}{\left(M_{l}^{-1} x\right)^{i}} \\
& \times \exp \left[-\frac{1}{2}\left(\ln \left(M_{l}^{-1} x\right)-\mu_{l}(t)\right)^{T}\left(\Sigma_{l}(t)\right)^{-1}\left(\ln \left(M_{l}^{-1} x\right)-\mu_{l}(t)\right)\right] \\
& \times \prod_{k=0}^{l-1}\left[\int_{0}^{\infty}(2 \pi)^{-n / 2}\left|\Sigma_{k}\left(t_{k+1}\right)\right|^{-1 / 2}\left|M_{k}^{-1}\right| \prod_{i=1}^{n} \frac{1}{\left(M_{k}^{-1}\left(x_{k+1} / z_{k+1}\right)\right)^{i}}\right. \\
& \quad \times \exp \left[-\frac{1}{2}\left(\ln \left(M_{k}^{-1}\left(\frac{x_{k+1}}{z_{k+1}}\right)\right)-\mu_{k}\left(t_{k+1}\right)\right)^{T}\left(\Sigma_{k}\left(t_{k+1}\right)\right)^{-1}\right. \\
&\left.\left.\quad \times\left(\ln \left(M_{k}^{-1}\left(\frac{x_{k+1}}{z_{k+1}}\right)\right)-\mu_{k}\left(t_{k+1}\right)\right)\right] g\left(z_{k+1}\right) \frac{1}{z_{k+1}^{n}} d z_{k+1}\right] .
\end{aligned}
$$

The conditional probability density function of $x(t)$ given in (4.16) is obtained by integrating (4.19) with respect to $x_{1}, x_{2}, \ldots$, and $x_{l}$. 
Now, we can derive the unconditional probability distribution of the solution process of the $n$-dimensional system given in the following proposition.

Proposition 4.6. Under assumptions (i)-(iii), the probability density function of the solution process $x(t)$ to the $n$-dimensional system (2.9) is given by

$$
\begin{gathered}
f_{x(t)}(x) \\
=\sum_{l=0}^{\infty}\left[\int_{0}^{t} \cdots \int_{0}^{t_{3}} \int_{0}^{t_{2}}\right. \\
\times\left[\int_{\mathbb{R}_{+}^{n}} \cdots \int_{\mathbb{R}_{+}^{n}}(2 \pi)^{-n / 2}\left|\Sigma_{l}(t)\right|^{-1 / 2}\left|M_{l}^{-1}\right| \prod_{i=1}^{n} \frac{1}{\left(M_{l}^{-1} x\right)^{i}}\right. \\
\times \exp \left[-\frac{1}{2}\left(\ln \left(M_{l}^{-1} x\right)-\mu_{l}(t)\right)^{T}\left(\Sigma_{l}(t)\right)^{-1}\left(\ln \left(M_{l}^{-1} x\right)-\mu_{l}(t)\right)\right] \\
\times \prod_{k=0}^{l-1}\left[\int_{0}^{\infty}(2 \pi)^{-n / 2}\left|\Sigma_{k}\left(t_{k+1}\right)\right|^{-1 / 2}\left|M_{k}^{-1}\right| \prod_{i=1}^{n} \frac{1}{\left(M_{k}^{-1}\left(x_{k+1} / z_{k+1}\right)\right)^{i}}\right. \\
\times \exp \left[-\frac{1}{2}\left(\ln \left(M_{k}^{-1}\left(\frac{x_{k+1}}{z_{k+1}}\right)\right)-\mu_{k}\left(t_{k+1}\right)\right)^{T}\left(\Sigma_{k}\left(t_{k+1}\right)\right)^{-1}\right. \\
\left.\times\left(\ln \left(M_{k}^{-1}\left(\frac{x_{k+1}}{z_{k+1}}\right)\right)-\mu_{k}\left(t_{k+1}\right)\right)\right] \\
\left.\times \prod_{i=1}^{l} \lambda\left(t_{i}\right) d t_{1} d t_{2} \cdots d t_{l} \exp \left[-\int_{0}^{t} \lambda(u) d u\right]\right]
\end{gathered}
$$

Proof. The proof follows the argument in Proposition 3.3 by incorporating the random jumps.

Remark 4.7. It is obvious that the result in Proposition 4.6 yields the one-dimensional result as a special case. As a result of this, the proof for Proposition 4.6 is considered to be an alternative proof of the one-dimensional result in Proposition 3.3.

\section{Probability Distribution of an Ornstein-Uhlenbeck Model with Jumps}

In this section, we will derive the probability distribution of an Ornstein-Uhlenbeck model with jumps described by system (2.12). To obtain the desired result, we need the following lemma which gives the probability distribution of an Ornstein-Uhlenbeck process which is the continuous dynamic between jumps in system (2.12). 
Lemma 5.1 (see $[18,29])$. The solution process $x(t)$ of the Ornstein-Uhlenbeck equation

$$
d x(t)=A x(t) d t+C d \bar{w}(t), \quad t \geq t_{0}, x\left(t_{0}\right)=x_{0}
$$

follows a multivariate normal distribution with mean $\mu(t)$ and covariance matrix $\Sigma(t)$, where

$$
\mu(t)=\exp ^{A\left(t-t_{0}\right)} x_{0}, \quad \Sigma(t)=\int_{t_{0}}^{t} e^{A(t-u)} V e^{A^{T}(t-u)} d u,
$$

and $V=C C^{T}$

Suppose that the number of jumps and the jump times are given, then, by applying the above lemma piecewisely, the following result gives the conditional probability density function of $x(t)$.

Lemma 5.2. Under the conditions $N(t)=l$, and $T_{1}=t_{1}, T_{2}=t_{2}, \ldots, T_{l}=t_{l}$, the solution process $x(t)$ for system (2.12) has a conditional probability density function as

$$
\begin{aligned}
f_{x(t) \mid N(t)=l, t_{1}, \ldots, t_{l}}(x)= & \int_{\mathbb{R}^{n}} \cdots \int_{\mathbb{R}^{n}} f_{x(t), x\left(t_{l}\right), \ldots, x\left(t_{1}\right) \mid N(t)=l, t_{1}, \ldots, t_{l}}\left(x, x_{l}, \ldots, x_{1}\right) d x_{l} \cdots d x_{1} \\
= & \int_{\mathbb{R}^{n}} \ldots \int_{\mathbb{R}^{n}}(2 \pi)^{-n / 2}\left|\Sigma_{l}(t)\right|^{-1 / 2} \exp \left[-\frac{1}{2}\left(x-\mu_{l}(t)\right)^{T}\left(\Sigma_{l}(t)\right)^{-1}\left(x-\mu_{l}(t)\right)\right] \\
\times & \quad \prod_{k=0}^{l-1}\left[\int_{0}^{\infty}(2 \pi)^{-n / 2}\left|\Sigma_{k}\left(t_{k+1}\right)\right|^{-1 / 2}\right. \\
& \quad \times \exp \left[-\frac{1}{2}\left(\frac{x_{k+1}}{z_{k+1}}-\mu_{k}\left(t_{k+1}\right)\right)^{T}\left(\Sigma_{k}\left(t_{k+1}\right)\right)^{-1}\left(\frac{x_{k+1}}{z_{k+1}}-\mu_{k}\left(t_{k+1}\right)\right)\right] \\
& \left.\times g\left(z_{k+1}\right) \frac{1}{z_{k+1}^{n}} d z_{k+1}\right] d x_{l} \cdots d x_{1},
\end{aligned}
$$

where $g$ is the common probability density function of $z_{k}, k=1,2, \ldots, l$.

Proof. As we noted before that the joint probability density function of $\left(x(t), x\left(t_{l}\right), \ldots, x\left(t_{1}\right)\right)$, under the conditions $N(t)=l$ and $T_{1}=t_{1}, T_{2}=t_{2}, \ldots, T_{l}=\mathrm{t}_{l}$, can be expressed as

$$
\begin{aligned}
& f_{x(t), x\left(t_{l}\right), \ldots, x\left(t_{1}\right) \mid N(t)=l, t_{1}, \ldots, t_{l}}\left(x, x_{l}, \ldots, x_{1}\right) \\
& \quad=f_{x(t) \mid x\left(t_{l}\right), N(t)=l, t_{1}, \ldots, t_{l}}(x) f_{x\left(t_{l}\right) \mid x\left(t_{l-1}\right), N(t)=l, t_{1}, \ldots, t_{l}}\left(x_{l}\right) \cdots f_{x\left(t_{1}\right) \mid N(t)=l, t_{1}, \ldots, t_{l}}\left(x_{1}\right),
\end{aligned}
$$


then, by applying the result in Lemma 5.1 on each interval between jumps $\left[t_{k}, t_{k+1}\right)$, we have, for $s \in\left[t_{k}, t_{k+1}\right)$,

$$
\begin{aligned}
f_{x(s) \mid x\left(t_{k}\right), N(t)=l, t_{1}, \ldots, t_{l}}(x) & =\phi\left(x ; \mu_{k}(s), \Sigma_{k}(s)\right) \\
& =(2 \pi)^{-n / 2}\left|\Sigma_{k}(s)\right|^{-1 / 2} \exp \left[-\frac{1}{2}\left(x-\mu_{k}(s)\right)^{T}\left(\Sigma_{k}(s)\right)^{-1}\left(x-\mu_{k}(s)\right)\right],
\end{aligned}
$$

where

$$
\mu_{k}(s)=\exp ^{A_{k}\left(s-t_{k}\right)} x_{k}, \quad \Sigma_{k}(s)=\int_{t_{k}}^{s} e^{A_{k}(s-u)} V_{k} e^{A_{k}^{T}(s-u)} d u,
$$

and $V_{k}=C_{k} C_{k}^{T}$. Then, for $k=0,1, \ldots, l-1$, consider that $x\left(t_{k+1}\right)=x\left(t_{k+1}^{-}\right) z_{k+1}$ as a product of two random variables where the first one has the probability density function given in (5.5), and $z_{k+1}$ is the random jump factor at time $t_{k+1}$. By the independence of $x\left(t_{k+1}^{-}\right)$and $z_{k+1}$, then

$$
\begin{aligned}
f_{x\left(t_{k+1}\right) \mid N(t)=l, t_{1}, \ldots, t_{l}}\left(x_{k+1}\right)= & \int_{0}^{\infty} f_{x\left(t_{k+1}^{-}\right) \mid N(t)=l, t_{1}, \ldots, t_{l}}\left(\frac{x_{k+1}}{z_{k+1}}\right) g\left(z_{k+1}\right) \frac{1}{z_{k+1}^{n}} d z_{k+1} \\
= & \int_{0}^{\infty}(2 \pi)^{-n / 2}\left|\Sigma_{k}\left(t_{k+1}\right)\right|^{-1 / 2} \\
& \times \exp \left[-\frac{1}{2}\left(\frac{x_{k+1}}{z_{k+1}}-\mu_{k}\left(t_{k+1}\right)\right)^{T}\left(\Sigma_{k}\left(t_{k+1}\right)\right)^{-1}\left(\frac{x_{k+1}}{z_{k+1}}-\mu_{k}\left(t_{k+1}\right)\right)\right] \\
& \times g\left(z_{k+1}\right) \frac{1}{z_{k+1}^{n}} d z_{k+1} .
\end{aligned}
$$

Then the conditional joint probability density function (5.4) can be written as

$$
\begin{aligned}
f_{x(t), x\left(t_{l}\right), \ldots, x\left(t_{1}\right) \mid N(t)=l, t_{1}, \ldots, t_{l}}\left(x, x_{l}, \ldots, x_{1}\right) \\
=(2 \pi)^{-n / 2}\left|\Sigma_{l}(t)\right|^{-1 / 2} \exp \left[-\frac{1}{2}\left(x-\mu_{l}(t)\right)^{T}\left(\Sigma_{l}(t)\right)^{-1}\left(x-\mu_{l}(t)\right)\right] \\
\times \prod_{k=0}^{l-1}\left[\int_{0}^{\infty}(2 \pi)^{-n / 2}\left|\Sigma_{k}\left(t_{k+1}\right)\right|^{-1 / 2}\right. \\
\quad \times \exp \left[-\frac{1}{2}\left(\frac{x_{k+1}}{z_{k+1}}-\mu_{k}\left(t_{k+1}\right)\right)^{T}\left(\Sigma_{k}\left(t_{k+1}\right)\right)^{-1}\left(\frac{x_{k+1}}{z_{k+1}}-\mu_{k}\left(t_{k+1}\right)\right)\right] \\
\left.\quad \times g\left(z_{k+1}\right) \frac{1}{z_{k+1}^{n}} d z_{k+1}\right] .
\end{aligned}
$$

The conditional probability density function of $x(t)$ given in (5.3) is obtained by integrating (5.8) with respect to $x_{1}, x_{2}, \ldots$, and $x_{l}$. 
Finally, following the same argument in Proposition 3.3, we present the unconditional probability distribution of the solution process of the Ornstein-Uhlenbeck models with jumps given in the following proposition.

Proposition 5.3. The probability density function of the solution process $x(t)$ of system (2.12) is given by

$$
\begin{aligned}
f_{x(t)}(x)=\sum_{l=0}^{\infty}\left[\int _ { 0 } ^ { t } \cdots \int _ { 0 } ^ { t _ { 3 } } \int _ { 0 } ^ { t _ { 2 } } \left[\int_{\mathbb{R}^{n}} \cdots \int_{\mathbb{R}^{n}}(2 \pi)^{-n / 2}\left|\Sigma_{l}(t)\right|^{-1 / 2}\right.\right. \\
\quad \times \exp \left[-\frac{1}{2}\left(x-\mu_{l}(t)\right)^{T}\left(\Sigma_{l}(t)\right)^{-1}\left(x-\mu_{l}(t)\right)\right] \\
\quad \times \prod_{k=0}^{l-1}\left[\int_{0}^{\infty}(2 \pi)^{-n / 2}\left|\Sigma_{k}\left(t_{k+1}\right)\right|^{-1 / 2}\right. \\
\quad \times \exp \left[-\frac{1}{2}\left(\frac{x_{k+1}}{z_{k+1}}-\mu_{k}\left(t_{k+1}\right)\right)^{T}\left(\Sigma_{k}\left(t_{k+1}\right)\right)^{-1}\left(\frac{x_{k+1}}{z_{k+1}}-\mu_{k}\left(t_{k+1}\right)\right)\right] \\
\left.\left.\quad \times g\left(z_{k+1}\right) \frac{1}{z_{k+1}^{n}} d z_{k+1}\right] d x_{l} \cdots d x_{1}\right] \prod_{i=1}^{l} \lambda\left(t_{i}\right) d t_{1} d t_{2} \cdots d t_{l} \\
\quad \times \exp \left[-\int_{0}^{t} \lambda(u) d u\right] .
\end{aligned}
$$

\section{Concluding Remarks}

In this work, the closed-form solutions of general linear non-homogeneous stochastic hybrid systems are obtained. The methods of finding probability density functions of closed-form solutions are initiated for the linear homogeneous system, and for system with drift and additive noise (Ornstein-Uhlenbeck system). This approach provides a procedure of finding the probability density functions without solving the Fokker-Planck equations. In fact, the Fokker-Planck equation corresponding to system (2.9) has state-dependent coefficients. For example, for $n=1$ the Fokker-Planck equation corresponding to system (2.9) even in the absence of discrete time interventions is given by

$$
\begin{aligned}
\frac{\partial f}{\partial t} & =-\frac{\partial}{\partial x}[A x f]+\frac{\partial^{2}}{\partial x^{2}}\left[B^{2} x^{2} f\right] \\
& =\left(2 B^{2}-A\right) f+\left(4 B^{2}-A\right) x \frac{\partial}{\partial x}+B^{2} x^{2} \frac{\partial^{2}}{\partial x^{2}} .
\end{aligned}
$$

As a result of this, equations of this type are not easily solvable in closed-form solutions. In future, we attempt to find the probability distribution of the solution of the general linear non-homogeneous system. In addition, by employing nonlinear transformation, we hope to develop probability distributions for nonlinear systems. 


\section{Acknowledgments}

This research was supported by the Mathematical Sciences Division, U.S. Army Research Office, Grant no. W911NF-07-1-0283. The authors would like to express their gratitude to the referee and the editor for their constructive, scientific thorough review and comments on the paper.

\section{References}

[1] M. H. A. Davis, "Piecewise-deterministic Markov processes: a general class of non-diffusion stochastic models," Journal of the Royal Statistical Society, vol. 46, no. 3, pp. 353-388, 1984.

[2] M. H. A. Davis, Markov Models and Optimization, Chapman and Hall, London, Uk, 1993.

[3] J. P. Hespanha, "A model for stochastic hybrid systems with application to communication networks," Nonlinear Analysis, vol. 62, no. 8, pp. 1353-1383, 2005.

[4] J. Hu, J. Lygeros, and S. Sastry, "Towards a theory of stochastic hybrid systems," Hybrid Systems: Computation and Control, vol. 1790, pp. 160-173, 2000.

[5] H. J. Kushner, Stochastic Stability and Control, Academic Press, New York, NY, USA, 1967.

[6] G. S. Ladde and V. Lakshmikantham, Random Differential Inequalities, Academic Press, New York, NY, USA, 1980.

[7] D. P. Siu and G. S. Ladde, "Stochastic hybrid system with non-homogeneous jumps," Nonlinear Analysis: Hybrid Systems, vol. 5, pp. 591-602, 2011.

[8] J. Chandra and G. S. Ladde, "Stability analysis of stochastic hybrid systems," International Journal of Hybrid Systems, vol. 4, pp. 179-198, 2004.

[9] A. Korzeniowski and G. S. Ladde, "Modeling hybrid network dynamics under random perturbations," Nonlinear Analysis: Hybrid Systems, vol. 3, no. 2, pp. 143-149, 2009.

[10] G. S. Ladde, "Hybrid dynamic inequalities and applications," Dynamic Systems and Applications, vol. 14, no. 3-4, pp. 481-513, 2005.

[11] G. Yin, Q. S. Song, and Z. Zhang, "Numerical solutions for jump-diffusions with regime switching," Stochastics: An International Journal of Probability and Stochastic Processes, vol. 77, no. 1, pp. 61-79, 2005.

[12] X. Mao and C. Yuan, Stochastic Differential Equations with Markovian Switching, Imperial College Press, London, UK, 2006.

[13] G. G. Yin and C. Zhu, Hybrid Switching Diffusions, Springer, New York, NY, USA, 2010.

[14] J. H. Dshalalow, S. Kim, and L. Tadj, "Hybrid queueing systems with hysteretic bilevel control policies," Nonlinear Analysis, vol. 65, no. 11, pp. 2153-2168, 2006.

[15] H. A. P. Blom, J. Krystul, and G. J. Bakker, "A particle system for safety verification of free flight in air traffic," in Proceedings of the 45th IEEE Conference on Decision and Control, pp. 1574-1579, San Diego, Calif, USA, December 2006.

[16] W. Glover and J. Lygeros, "A stochastic hybrid model for air traffic control simulation," Lecture Notes in Computer Science, vol. 2993, pp. 372-386, 2004.

[17] A. Dassios and P. Embrechts, "Martingales and insurance risk," Stochastic Models, vol. 5, no. 2, pp. 181-217, 1989.

[18] H. Risken, The Fokker-Planck Equation: Methods of Solutions and Applications, Springer, New York, NY, USA, 1989.

[19] A. G. Ladde and G. S. Ladde, Introduction to Differential Equations II: Stochastic Modeling, Methods and Analysis, World Scientific Publishing Company, In press.

[20] B. Øksendal, Stochastic Differential Equations, Springer, Berlin, Germany, 6th edition, 2003.

[21] R. Rosen, Dynamical System Theory in Biology, vol. I, Wiley-Interscience, New York, NY, USA, 1970.

[22] D. D. Siljak, Large-Scale Dynamic Systems, North-Holland, New York, NY, USA, 1978.

[23] J. R. Movellan, “Tutorial on Stochastic Differential Equations," 2006, MPLab Tutorials Version 06.1.

[24] D. R. Cox and P. A. W. Lewis, The Statistical Analysis of Series of Events, Methuen, London, UK, 1966.

[25] M. J. Crowder, A. C. Kimber, R. L. Smith, and T. J. Sweeting, Statistical Analysis of Reliability Data, Chapman and Hall, London, UK, 1991.

[26] V. K. Rohatgi and A. K. Saleh, An Introduction to Probability and Statistics, Wiley, New York, NY, USA, 2nd edition, 2001.

[27] R. A. Horn and C. R. Johnson, Matrix Analysis, Cambridge University Press, New York, NY, USA, 1985. 
[28] J. Stachurski, "Log-linearization of perturbed dynamical systems, with applications to optimal growth," Research Paper 788, Department of Economics, University of Melbourne, 2001.

[29] A. Meucci, "Review of statistical arbitrage, cointegration, and multivariate Ornstein-Uhlenbeck," 2010, http:/ / ssrn.com/abstract=1404905 . 


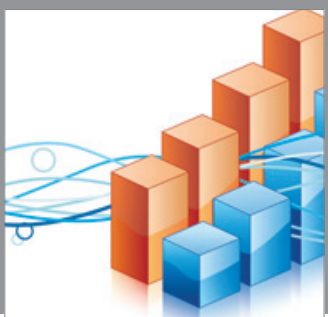

Advances in

Operations Research

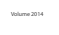

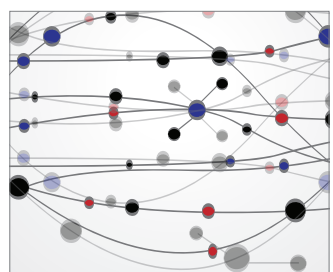

\section{The Scientific} World Journal
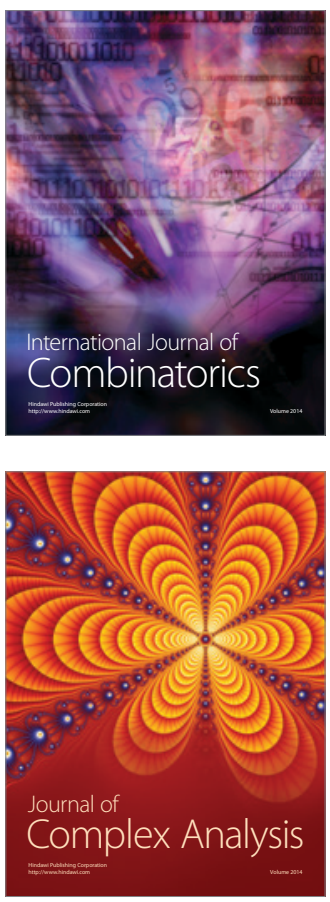

International Journal of

Mathematics and

Mathematical

Sciences
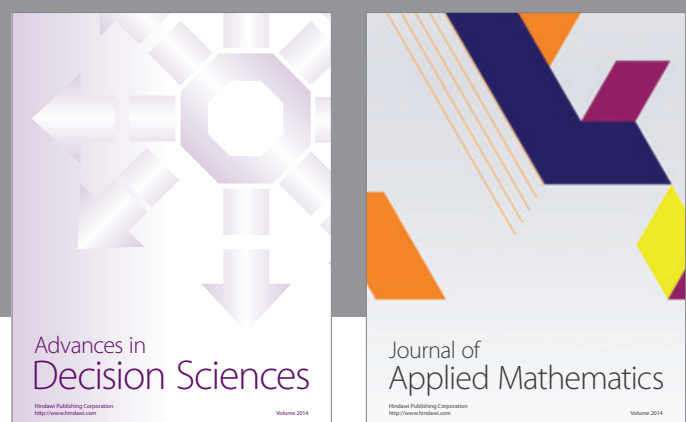

Journal of

Applied Mathematics


Submit your manuscripts at http://www.hindawi.com
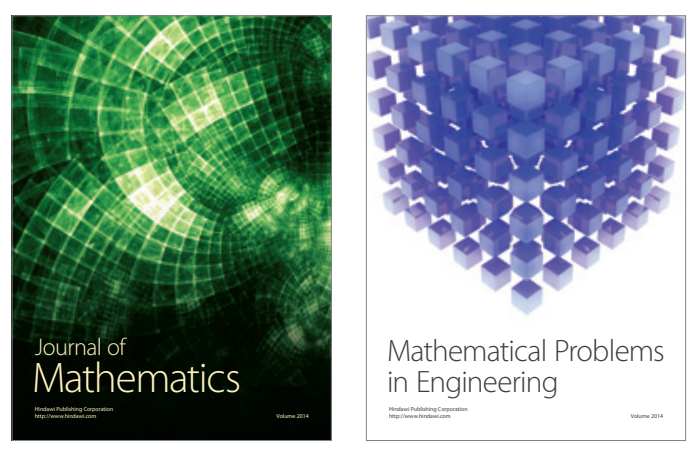

Mathematical Problems in Engineering
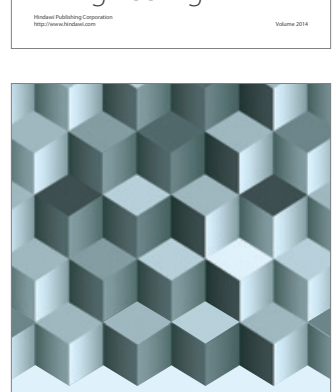

Journal of

Function Spaces
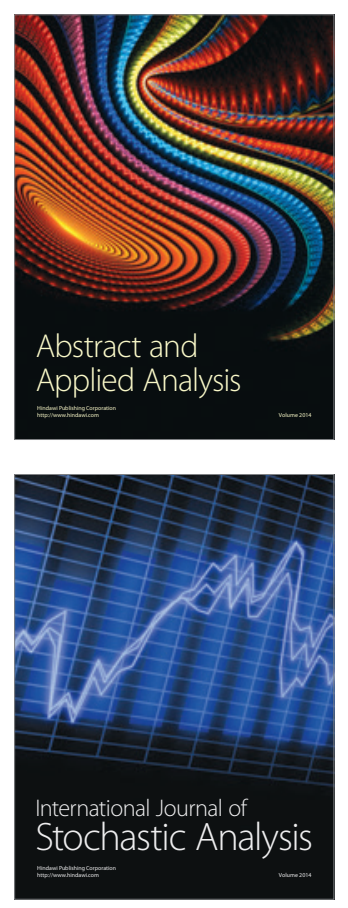

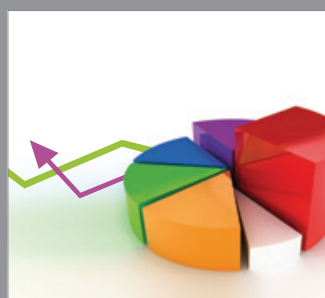

ournal of

Probability and Statistics

Promensencen
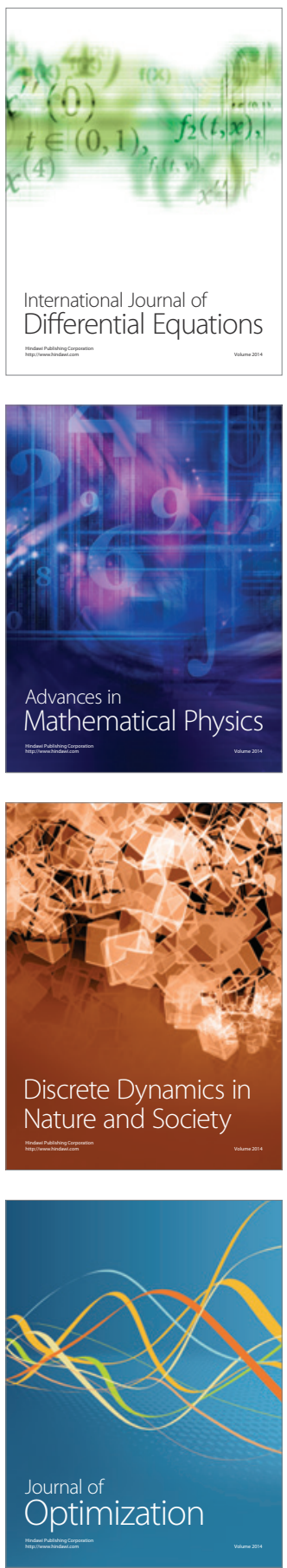